\title{
The effects of LIGO detector noise on a 15-dimensional Markov-chain Monte-Carlo analysis of gravitational-wave signals
}

\author{
V. Raymond ${ }^{1}$, M.V. van der Sluys ${ }^{2}$, I. Mandel ${ }^{1}$, V. Kalogera ${ }^{1}$, \\ C. Röver ${ }^{3}$, N. Christensen ${ }^{4}$ \\ ${ }^{1}$ Dept. of Physics \& Astronomy, Northwestern University, 2131 Tech Drive, \\ Evanston IL, 60208, USA \\ ${ }^{2}$ Dept. of Physics, University of Alberta, 11322 - 89 Avenue, Edmonton AB, T6G \\ 2G7, Canada \\ ${ }^{3}$ Max-Planck-Institut für Gravitationsphysik, Callinstraße 38, 30167 Hannover, \\ Germany \\ ${ }^{4}$ Physics \& Astronomy Dept., Carleton College, One North College Street, \\ Northfield MN, 55057, USA \\ E-mail: vivien@u.northwestern.edu
}

\begin{abstract}
Gravitational-wave signals from inspirals of binary compact objects (black holes and neutron stars) are primary targets of the ongoing searches by ground-based gravitational-wave (GW) interferometers (LIGO, Virgo, and GEO-600). We present parameter-estimation results from our Markov-chain Monte-Carlo code SPINSPIRAL on signals from binaries with precessing spins. Two data sets are created by injecting simulated GW signals into either synthetic Gaussian noise or into LIGO detector data. We compute the 15-dimensional probability-density functions (PDFs) for both data sets, as well as for a data set containing LIGO data with a known, loud artefact ("glitch"). We show that the analysis of the signal in detector noise yields accuracies similar to those obtained using simulated Gaussian noise. We also find that while the Markov chains from the glitch do not converge, the PDFs would look consistent with a GW signal present in the data. While our parameter-estimation results are encouraging, further investigations into how to differentiate an actual GW signal from noise are necessary.
\end{abstract}




\section{Introduction}

Among the sources of gravitational waves (GWs), inspiralling binary systems of compact objects, neutron stars (NSs) and/or black holes (BHs) in the mass range $\sim 1 M_{\odot}-100 M_{\odot}$ stand out as likely to be detected and relatively easy to model. For ground-based laser interferometers currently in operation (Cutler \& Thorne 2002), LIGO (Abbott et al. 2009a), Virgo (Acernese et al. 2008) and GEO-600 (Willke et al. 2004), the current detection-rate estimates for BH-NS binaries range from $2 \times 10^{-4}$ to $0.2 \mathrm{yr}^{-1}$ for first-generation instruments (e.g. O'Shaughnessy et al. 2008, Abadie et al. 2010). Although the estimates are quite uncertain, detection rates are expected to increase with the upgrade to Enhanced LIGO/Virgo, up to $\sim 40 \mathrm{yr}^{-1}$ with Advanced LIGO/Virgo.

The detection of a gravitational-wave event is challenging and will be a rewarding achievement by itself. After such a detection, measurement of source properties holds major promise for improving our astrophysical understanding and requires reliable methods for parameter estimation. This is a complicated problem, because of the large number of parameters (15 for spinning compact objects in a quasi-circular orbit) and the degeneracies between them (Raymond et al. 2009), the significant amount of structure in the parameter space, and the particularities of the detector noise.

In this paper we use an example to illustrate the capabilities of our Markovchain Monte-Carlo (MCMC) algorithm SPINsPIRAL (Van der Sluys et al. 2008a) for parameter estimation of binary inspirals with two spinning components, using groundbased GW interferometers. In these proceedings we focus on the effects of using LIGO detector data versus synthetic Gaussian noise. Earlier studies (e.g. Jaranowski \& Krolak 1994, Cutler \& Flanagan 1994, Poisson \& Will 1995, Van den Broeck \& Sengupta 2007) computed the potential accuracy of parameter estimation (e.g. by using the Fisher matrix), but without performing a parameter estimation in practice. Also, Röver et al. (2006, 2007), Veitch \& Vecchio (2008b, 2008a, 2009) explored parameter estimation for binaries without spins, described by nine parameters.

We present the gravitational-wave template used for this study in section 2, and the Bayesian framework we employ here in section [3. In section 4.1 we describe the three data sets that we analyse in this study; a simulated GW signal injected into synthetic Gaussian noise, a GW signal injected into LIGO detector data and a raw LIGO data set containing a known artefact of terrestrial origin ("glitch"). We describe the details of the MCMC simulations in section 4.2. The analyses of the first two data sets are compared in section 4.3, and we present our results on the glitch in section 4.4.

\section{Gravitational-wave signal and observables}

We analyse the signal produced during the inspiral phase of two compact objects of masses $M_{1,2}$ in quasi-circular orbit. We focus on a black-hole binary system with $M_{1}=10 M_{\odot}$ and $M_{2}=1.4 M_{\odot}$, where unlike in some of our previous studies (e.g. Van der Sluys et al. 2008b), we do not ignore the second spin to explore the single spin 
approximation. During the orbital inspiral, the general-relativistic spin-orbit and spinspin coupling (dragging of inertial frames) cause the binary's orbital plane to precess and introduce amplitude and phase modulations of the observed gravitational-wave signal (Apostolatos et al. 1994).

A circular binary inspiral with both compact objects spinning is described by a 15-dimensional parameter vector $\vec{\lambda} \in \Lambda$. Our choice of independent parameters with respect to a fixed geocentric coordinate system is:

$$
\begin{aligned}
\vec{\lambda}= & \left\{\mathcal{M}, \eta, \log d_{\mathrm{L}}, t_{\mathrm{c}}, \phi_{\mathrm{c}}, \alpha, \cos \delta, \sin \iota, \psi,\right. \\
& \left.a_{\mathrm{spin} 1}, \cos \theta_{\mathrm{spin} 1}, \phi_{\mathrm{spin} 1}, a_{\mathrm{spin} 2}, \cos \theta_{\mathrm{spin} 2}, \phi_{\mathrm{spin} 2}\right\},
\end{aligned}
$$

where $\mathcal{M}=\frac{\left(M_{1} M_{2}\right)^{3 / 5}}{\left(M_{1}+M_{2}\right)^{1 / 5}}$ and $\eta=\frac{M_{1} M_{2}}{\left(M_{1}+M_{2}\right)^{2}}$ are the chirp mass and symmetric mass ratio, respectively; $d_{\mathrm{L}}$ is the luminosity distance to the source; $\phi_{\mathrm{c}}$ is an integration constant that specifies the GW phase at the time of coalescence $t_{\mathrm{c}}$, defined with respect to the centre of the Earth; $\alpha$ (right ascension) and $\delta$ (declination) identify the source position in the sky; $\iota$ defines the inclination of the binary with respect to the line of sight; and $\psi$ is the polarisation angle of the waveform. The spins are specified by $0 \leq a_{\operatorname{spin}_{1,2}} \equiv S_{1,2} / M_{1,2}^{2} \leq 1$ as the dimensionless spin magnitude, and the angles $\theta_{\text {spin } 1,2}, \phi_{\text {spin } 1,2}$ for their orientations.

Given a network comprising $n_{\text {det }}$ detectors, the data collected at the $a$-th instrument $\left(a=1, \ldots, n_{\text {det }}\right)$ is given by $x_{a}(t)=n_{a}(t)+h_{a}(t ; \vec{\lambda})$, where $h_{a}(t ; \vec{\lambda})=$ $F_{a,+}(t, \alpha, \delta, \psi) h_{a,+}(t ; \vec{\lambda})+F_{a, \times}(t, \alpha, \delta, \psi) h_{a, \times}(t ; \vec{\lambda})$ is the GW strain at the detector (see Eqs. $2-5$ in Apostolatos et al. 1994) and $n_{a}(t)$ is the detector noise. The astrophysical signal is given by the linear combination of the two independent polarisations $h_{a,+}(t ; \vec{\lambda})$ and $h_{a, \times}(t ; \vec{\lambda})$ weighted by the antenna beam patterns $F_{a,+}(t, \alpha, \delta, \psi)$ and $F_{a, \times}(t, \alpha, \delta, \psi)$.

The waveform we use includes terms up to 3.5-post-Newtonian (pN) order in phase and uses Newtonian amplitudes, with spin effects up to 2.5-pN in phase. We generate the waveform templates using the routine LALGenerateInspiral() with the approximant SpinTaylor from the injection package in the LSC Algorithm Library (LAL) (LIGO Scientific Collaboration 2007), which closely follows the first section of Buonanno et al. (2003).

\section{Parameter estimation: Methods}

In our Bayesian analysis we use MCMC methods to determine the multi-dimensional posterior probability-density function (PDF) of the unknown parameter vector $\vec{\lambda}$ in equation 1, given the data sets $x_{a}$ collected by a network of $n_{\text {det }}$ detectors, a model $M$ of the waveform and the prior $p(\vec{\lambda})$ on the parameters. Our priors are uniform in the parameters of Eq.1 (see Van der Sluys et al. (2008a) for details). One can compute the probability density via Bayes' theorem

$$
p\left(\vec{\lambda} \mid x_{a}, M\right)=\frac{p(\vec{\lambda} \mid M) p\left(x_{a} \mid \vec{\lambda}, M\right)}{p\left(x_{a} \mid M\right)},
$$


where

$$
\mathcal{L} \equiv p\left(x_{a} \mid \vec{\lambda}, M\right) \propto \exp \left(<x_{a}\left|h_{a}(\vec{\lambda})>-\frac{1}{2}<h_{a}(\vec{\lambda})\right| h_{a}(\vec{\lambda})>\right)
$$

is the likelihood function, which measures how well the data fits the model $M$ for the parameter vector $\vec{\lambda}$. The term $p\left(x_{a} \mid M\right)$ is the marginal likelihood or evidence. In the previous equation

$$
<x \mid y>=4 R e\left(\int_{f_{\text {low }}}^{f_{\text {high }}} \frac{\tilde{x}(f) \tilde{y}^{*}(f)}{S_{a}(f)} \mathrm{d} f\right)
$$

is the overlap of signals $x$ and $y, \tilde{x}(f)$ is the Fourier transform of $x(t)$, and $S_{a}(f)$ is the noise power-spectral density in detector $a$. The likelihood computed for the injection parameters $\mathcal{L}_{\text {inj }}=p\left(x_{a} \mid \vec{\lambda}_{\text {inj }}, M\right)$ is then a random variable that depends on the particular noise realisation $n_{a}$ in the data $x_{a}=h\left(\vec{\lambda}_{\text {inj }}\right)+n_{a}$. The injection parameters are the parameters of the waveform template added to the noise. We define the signal-to-noise ratio (SNR) of the injection to be:

$$
\mathrm{SNR}=\frac{<x \mid h\left(\vec{\lambda}_{\text {inj }}\right)>}{\sqrt{<h\left(\vec{\lambda}_{\text {inj }}\right) \mid h\left(\vec{\lambda}_{\text {inj }}\right)>}} .
$$

From here on, we use the expected value of the SNR, which is equal to the square root of twice the expectation value of $\log \mathcal{L}_{\text {inj }}$ :

$$
\mathrm{SNR}=\sqrt{<h\left(\vec{\lambda}_{\text {inj }}\right) \mid h\left(\vec{\lambda}_{\text {inj }}\right)>}
$$

To combine observations from a network of detectors with uncorrelated noise realisations (this is the case in this paper as we use two non-co-located detectors) we have the likelihood $p(\vec{x} \mid \vec{\lambda}, M)=\prod_{a=1}^{n_{\text {det }}} p\left(x_{a} \mid \vec{\lambda}, M\right)$, for $\vec{x} \equiv\left\{x_{a}: a=1, \ldots, n_{\operatorname{det}}\right\}$ and

$$
p(\vec{\lambda} \mid \vec{x}, M)=\frac{p(\vec{\lambda} \mid M) p(\vec{x} \mid \vec{\lambda}, M)}{p(\vec{x} \mid M)} .
$$

The numerical computation of the PDF involves the evaluation of a large multimodal, multi-dimensional integral. Markov-chain Monte-Carlo (MCMC) methods (e.g. Gilks et al. 1996, Gelman et al. 1997, and references therein) have proved to be especially effective in tackling this numerical problem. We developed an adaptive (see Figueiredo \& Jain 2002, Atchadé \& Rosenthal 2005) MCMC algorithm to explore the parameter space $\Lambda$ efficiently while requiring the least amount of tuning for the specific signal analysed; the code is an extension of the one developed by some of the authors to explore MCMC methods for binaries without spin (Röver et al. 2006, Röver et al. 2007). We implemented parallel tempering (Hukushima \& Nemoto 1996, Hansmann 1997, Röver 2007) to improve the sampling. It consists of running several MCMC chains in parallel, each with a different "temperature", which can swap parameters under certain conditions. Only the $T=1$ chain is currently used for post-processing.

In Eq. 7 we applied Bayes' theorem to obtain the probability of a specific parameter vector value $(\vec{\lambda})$ given the observed data $\vec{x}$ and the model $M$. The theorem can also be applied to compute the probability of a specific model $M_{i}$ given the observed data:

$$
p\left(M_{i} \mid \vec{x}\right)=\frac{p\left(M_{i}\right) p\left(\vec{x} \mid M_{i}\right)}{p(\vec{x})} .
$$


We compare the two models $M_{i}$ and $M_{j}$ by computing the odds ratio:

$$
O_{i, j}=\frac{p\left(M_{i} \mid \vec{x}\right)}{p\left(M_{j} \mid \vec{x}\right)}=\frac{p\left(M_{i}\right) p\left(\vec{x} \mid M_{i}\right)}{p\left(M_{j}\right) p\left(\vec{x} \mid M_{j}\right)}=\frac{p\left(M_{i}\right)}{p\left(M_{j}\right)} B_{i, j},
$$

where

$$
B_{i, j}=\frac{p\left(\vec{x} \mid M_{i}\right)}{p\left(\vec{x} \mid M_{j}\right)}
$$

is the Bayes factor of the two models, and we recognise the evidence $p\left(\vec{x} \mid M_{i}\right)$ from Eq.7. The evidence must be marginalised over the parameters of the model in order to compute the Bayes factor:

$$
p\left(\vec{x} \mid M_{i}\right)=\int_{\Lambda} p\left(\vec{\lambda} \mid M_{i}\right) p\left(\vec{x} \mid \vec{\lambda}, M_{i}\right) \mathrm{d} \vec{\lambda}
$$

There are existing algorithms dedicated to the computation of this integral, and of the Bayes factor. For instance, nested sampling (Skilling 2006) has been shown to be very efficient in the case of non-spinning gravitational-wave sources (Veitch \& Vecchio 2009), and can in addition be used to produce PDFs of the parameters. As a by-product of the exploration of the parameter space with MCMC, it is possible to compute the evidences of the models used. We have implemented the harmonic-mean method (Newton \& Raftery 1994), in which the evidence is approximated by:

$$
p\left(\vec{x} \mid M_{i}\right) \approx \sum_{k=1}^{N} p\left(\vec{\lambda}_{k} \mid M_{i}\right) p\left(\vec{x} \mid \vec{\lambda}_{k}, M_{i}\right) V_{\vec{\lambda}_{k}}
$$

where $\left\{\vec{\lambda}_{k}: k=1, \ldots, N\right\}$ is the set of $N$ points sampled by the MCMC, and $V_{\vec{\lambda}_{k}}$ is the volume of parameter space associated with the point $\vec{\lambda}_{k}$. Since the MCMC algorithm samples according to the posterior (and, up to a proportionality constant, converges towards posterior PDF), the density of points in the chain at a certain location $\vec{\lambda}_{k}$ in the parameter space $\Lambda$ will become proportional to the posterior for large $N$. It follows that

$$
\lim _{N \rightarrow \infty} V_{\vec{\lambda}_{k}}=\frac{\alpha_{i}}{p\left(\vec{\lambda}_{k} \mid M_{i}\right) p\left(\vec{x} \mid \vec{\lambda}_{k}, M_{i}\right)}
$$

with $\alpha_{i}$ a proportionality constant. We then have $p\left(\vec{x} \mid M_{i}\right) \approx \sum_{k=1}^{N} \alpha_{i}=N \alpha_{i}$, and obtain the estimate for $\alpha_{i}$ by considering the whole parameter space volume $V_{t}$ :

$$
V_{t} \approx \sum_{k=1}^{N} V_{\vec{\lambda}_{k}}=\sum_{k=1}^{N} \frac{\alpha_{i}}{p\left(\vec{\lambda}_{k} \mid M_{i}\right) p\left(\vec{x} \mid \vec{\lambda}_{k}, M_{i}\right)} .
$$

Finally,

$$
p\left(\vec{x} \mid M_{i}\right) \approx N V_{t}\left[\sum_{k=1}^{N} \frac{1}{p\left(\vec{\lambda}_{k} \mid M_{i}\right) p\left(\vec{x} \mid \vec{\lambda}_{k}, M_{i}\right)}\right]^{-1},
$$

which is the harmonic mean of the posterior values sampled by the MCMC. The issue with this method is that it gives too much weight to low-posterior points, which lie in a part of the parameter space that is badly sampled, by design, by the MCMC. The estimate of the evidence is then very sensitive to the quality of the sampling of a 
particular run. We are looking into other algorithms in order to remedy this problem, e.g. by using the higher-temperature chains produced by parallel tempering (Earl \& Deem 2005) (we currently use the $T=1$ chain only), or by using a well sampled subset of points (Van Haasteren 2009) to estimate the probability constant $\alpha_{i}$. A summary of the methods used in our MCMC code was published in Van der Sluys et al. (2008a); a more complete technical description of the SPINSPIRAL code will be available in (Van der Sluys \& al. in preparation).

\section{Parameter estimation: Results}

\subsection{Data sets}

For these proceedings, we analyse three different data sets, each containing the data for the 4-km LIGO detectors at Hanford (H1) and Livingston (L1):

DS1: a coherent software injection with a total SNR of 11.3 into synthetic Gaussian, stationary noise, simulated for the $\mathrm{H} 1$ and L1 detectors;

DS2: a coherent software injection of the same signal, with a total SNR of 11.3, into "quiet" LIGO detector data from H1 and L1;

DS3: raw LIGO data from $\mathrm{H} 1$ and L1, containing a known, coincident glitch of seismic origin, with a total SNR of 11.3.

For the data sets DS1 and DS2, the injected signal is that of a $10 M_{\odot}$ spinning $\mathrm{BH}$ and a $1.4 M_{\odot}$ spinning NS in an inspiralling binary system. A low-mass Compact Binary Coalescence Group search (Abbott et al. 2009b) does not produce a GW trigger for the data segment DS2; hence we designate it "quiet". The distance of each of the injections is scaled to obtain an SNR of 11.3, equal to that of the glitch in DS3, but computed with different waveforms: a SpinTaylor waveform (see section2) for DS1 and DS2, and a non-spinning, 2-pN waveform (see section 4.4) for DS3. The other parameters of the injection are:

$$
\begin{aligned}
\vec{\lambda}= & \left\{\mathcal{M}=2.99 M_{\odot}, \eta=0.107, d_{\mathrm{L}}, t_{\mathrm{c}}, \phi_{\mathrm{c}}=85.9^{\circ}, \alpha=17.4 h, \delta=61.6^{\circ},\right. \\
& i=52.8^{\circ}, \psi=11.6^{\circ}, a_{\mathrm{spin} 1}=0.6, \theta_{\mathrm{spin} 1}=78.5^{\circ}, \phi_{\mathrm{spin} 1}=63.0^{\circ}, \\
& \left.a_{\mathrm{spin} 2}=0.4, \theta_{\mathrm{spin} 2}=120.0^{\circ}, \phi_{\mathrm{spin} 2}=315.1^{\circ}\right\},
\end{aligned}
$$

where we assigned a spin of 0.4 to the neutron star, which is higher than astrophysically plausible, for testing purposes only. In DS3, no signal is injected. For our analyses, we use the data of both 4-km LIGO detectors H1 and L1.

\section{2. $M C M C$ simulations}

The MCMC analysis that we carry out on each data set consists of 10 independent Markov chains, each with a length of about a million iterations and composed of 5 chains at different temperatures for parallel tempering. From now on, we will refer 
to the $T=1$ chain as the chain, since the hotter chains were not used in the postprocessing. The part of the chains that is analysed is that after the burn-in period (see e.g. Gilks et al. 1996), the length of which is determined automatically as follows: we determine the absolute maximum likelihood $\log \left(\mathcal{L}_{\max }\right)$, defined as the highest value for $\log [p(\vec{x} \mid \vec{\lambda}, M)]$ obtained over the ensemble of parameter sets $\vec{\lambda}$ in any of our individual Markov chains. Then for each chain we include all the iterations after the chain reaches a likelihood value of $\log \left(\mathcal{L}_{\max }\right)-2$ for the first time. This results in a convergence test as well, since some of the independent chains may not reach this threshold value. Typically, we demand that more than $50 \%$ of our chains meet this condition before we consider the MCMC run as converged, although we consider results as robust if they have a convergence rate of $80 \%$ or more. This convergence test is a measure of the quality of our sampling in a given number of iterations. All our Markov chains start at values that are randomly offset from the injection values. The starting values for $\mathcal{M}$ and $t_{\mathrm{c}}$ are drawn from a Gaussian distribution centred on the injection value, with a standard deviation of $0.025 M_{\odot}$ and $10 \mathrm{~ms}$ respectively. In real analysis, the two Gaussian distributions are centred on the values from the template bank based search of the Compact Binary Coalescence group (Abbott et al. 2009b) which will have triggered the MCMC followup. The other thirteen parameters are drawn uniformly from their allowed ranges. SPINSPIRAL needs to run for typically a few days in order to show the first results and a week or two to accumulate a sufficient number of iterations for good statistics, each chain using a single $2.8 \mathrm{GHz}$ CPU.

\subsection{Analysis of data sets DS1 and DS2}

We analysed the data sets DS1 and DS2 as described in section 4.1 and the results of both analyses passed the convergence test described in section 4.2 with convergence rates of $70 \%$ and $80 \%$, respectively. The resulting one-dimensional marginalised PDFs from both analyses are shown in figure 1.

Table 1 shows the median and the width of the 95\%-probability ranges for each parameter. The differences we find between the results for DS1 and DS2 may be attributed to the particular noise realisations in this example, and most parameters yield similar PDFs and accuracies.

The PDFs of the parameters that describe the spin of the NS follow the prior distributions in both runs. This justifies ignoring the NS spin (by fixing $a_{\text {spin2 }}$ to 0.0 in the recovery template) for this mass ratio (Van der Sluys et al. 2008b). For each of the two data sets, DS1 and DS2, we computed the Bayes factor to compare the evidence for the following two models: $M_{1}$ : a $3.5-\mathrm{pN}$ inspiral waveform embedded in Gaussian noise, and $M_{2}$ : Gaussian noise only. The values are listed in table 2. In both cases, the Bayes factor is large, providing strong evidence for a GW signal in the data. The difference in Bayes factor between DS1 and DS2 is attributed to an inherent spread due to different noise realisations, and the uncertainties of our method to estimate the Bayes factor (section 3). The results in this section show an illustrative example, but 

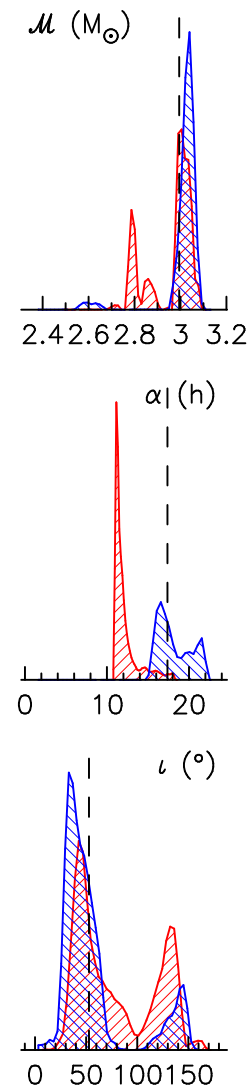
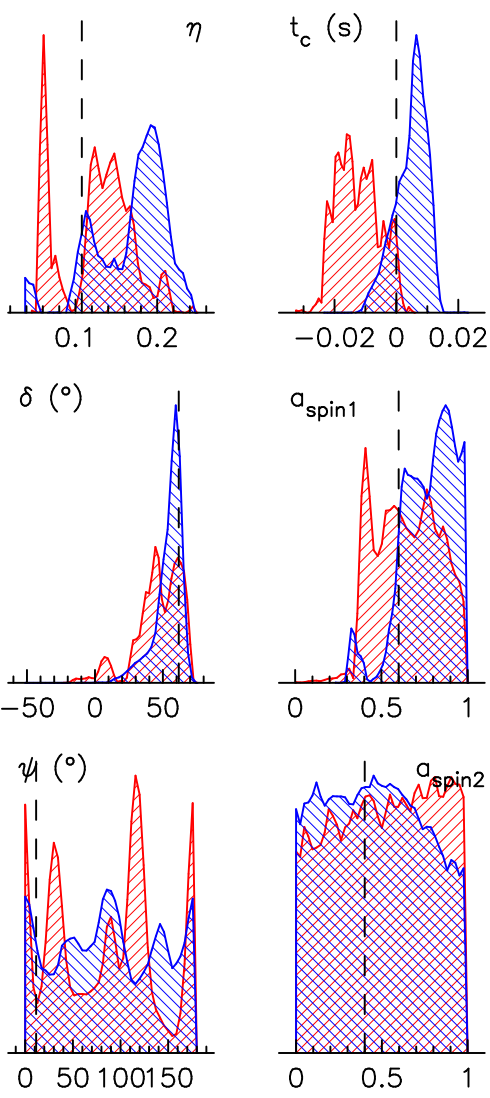
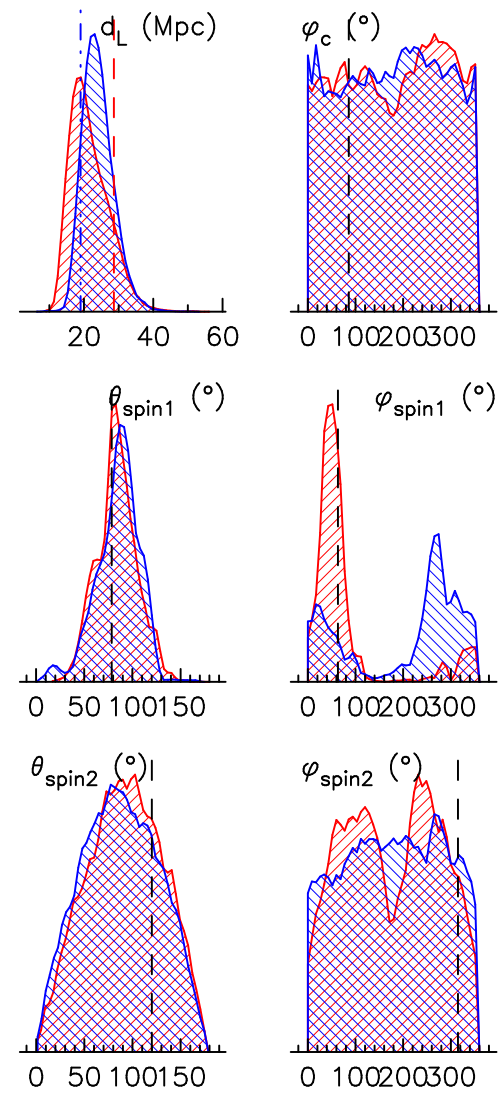

Figure 1: One-dimensional marginalised PDFs for all 15 parameters from our analysis of data sets DS1 (hatched upward; red in the online colour version) and DS2 (hatched downward; blue in the online colour version). The vertical dashed lines mark the injection values.

cannot be used to draw firm conclusions. However, it is clear that they warrant a larger, systematic study of these phenomena with the methods described here.

\subsection{Analysis of data sets DS2 and DS3}

On November 2nd 2006, seismic activity at Hanford and Livingston resulted in a coincident "glitch" in the data from the H1 and L1 LIGO detectors. These glitches were recovered by the Compact Binary Coalescence detection pipeline at an SNR of 11.3, using non-spinning, stationary-phase-approximation templates, Newtonian in amplitude and 2.0-pN in phase (Abbott et al. 2009b). We defined the corresponding data set as DS3 in section 4.1 and analysed the data as if it had yielded a GW trigger. The convergence test from section 4.2 yields a $20 \%$ convergence rate, which results in our rejection of the results as not converged. However, when we nevertheless construct the marginalised one-dimensional PDFs from the data of the two converged chains (because of the small number of data points, the resulting PDFs may not be very accurate), they are similar in appearance to those from DS2 (see figure 2). The Bayes factors in table 2 even suggest that the data set DS3 is more consistent with containing a GW signal than DS2 
Table 1: Median and width of the 95\%-probability ranges for each parameter of the analyses of data sets DS1 and DS2. The column recovered indicates whether or not the $95 \%$ range includes the injection value.

\begin{tabular}{l|l|lll|lll}
\hline & & \multicolumn{3}{|c|}{ DS1 (synthetic noise) } & \multicolumn{3}{c}{ DS2 (detector noise) } \\
& injection & median & $95 \%$ width & recovered & median & $95 \%$ width & recovered \\
\hline $\mathcal{M}\left(M_{\odot}\right)$ & 2.99 & 3.006 & 0.294 & yes & 3.041 & 0.122 & yes \\
$\eta$ & 0.107 & 0.133 & 0.145 & yes & 0.183 & 0.144 & yes \\
$d_{\mathrm{L}}(\mathrm{Mpc})$ & 28.615 & 21.240 & 20.764 & yes & 24.144 & 17.238 & yes \\
$t_{\mathrm{c}}(\mathrm{s})$ & 0.000 & -0.013 & 0.024 & yes & 0.006 & 0.019 & yes \\
$\phi_{\mathrm{c}}\left({ }^{\circ}\right)$ & 85.944 & 189.745 & 342.398 & yes & 185.482 & 343.175 & yes \\
$\alpha(\mathrm{h})$ & 17.380 & 11.684 & 5.349 & no & 17.786 & 6.320 & yes \\
$\delta\left(^{\circ}\right)$ & 61.642 & 49.326 & 64.346 & yes & 58.390 & 39.796 & yes \\
$i\left(^{\circ}\right)$ & 52.753 & 67.056 & 110.735 & yes & 46.850 & 122.787 & yes \\
$\psi\left(^{\circ}\right)$ & 11.459 & 93.162 & 176.358 & yes & 88.706 & 173.869 & yes \\
$a_{\text {spin1 }}$ & 0.600 & 0.658 & 0.594 & yes & 0.804 & 0.478 & yes \\
$\theta_{\text {spin1 }}\left({ }^{\circ}\right)$ & 78.463 & 85.490 & 83.110 & yes & 89.225 & 85.787 & yes \\
$\phi_{\text {spin1 }}\left({ }^{\circ}\right)$ & 63.025 & 57.171 & 335.592 & yes & 263.014 & 345.700 & yes \\
$a_{\text {spin2 }}$ & 0.400 & 0.532 & 0.945 & yes & 0.475 & 0.940 & yes \\
$\theta_{\text {spin2 }}\left({ }^{\circ}\right)$ & 120.000 & 94.687 & 150.544 & yes & 89.406 & 146.101 & yes \\
$\phi_{\text {spin2 }}\left(^{\circ}\right)$ & 315.127 & 181.959 & 327.603 & yes & 184.681 & 339.071 & yes \\
$M_{1}\left(M_{\odot}\right)$ & 10.002 & 8.533 & 8.849 & yes & 6.421 & 6.536 & yes \\
$M_{2}\left(M_{\odot}\right)$ & 1.400 & 1.598 & 1.277 & yes & 2.036 & 1.564 & yes \\
\hline
\end{tabular}

Table 2: Bayes factors $B_{1,2}$ between the models $M_{1}$ : a $3.5-\mathrm{pN}$ inspiral waveform embedded in Gaussian noise, and $M_{2}$ : Gaussian noise only (section 4.3) for data sets DS1 and DS2 (see section 4.1).

\begin{tabular}{cccc}
\hline & DS1 (Gaussian noise) & DS2 (detector data) & DS3 (glitch) \\
\hline $\log _{e} B_{1,2}$ & 52.9 & 43.5 & 68.5 \\
\hline
\end{tabular}

(with the caveat that the SNRs of DS2 and DS3 were not computed the same way). On the other hand, the low value for the median of $\eta(0.05)$ corresponds to a mass ratio of 18 , which is near the limit of the regime where post-Newtonian expansions are valid. In particular, a small value for eta suggests a slow frequency evolution which may indicate a spike in the frequency spectrum that dominates the signal. In addition, we find that the sky map for DS3 does not display the (parts of a) sky ring that is expected for an analysis using two non-co-located detectors (see e.g. Raymond et al. 2009). These results indicate that we should thoroughly verify our tests, such as the convergence criterion described here, using a large number of different glitches. 


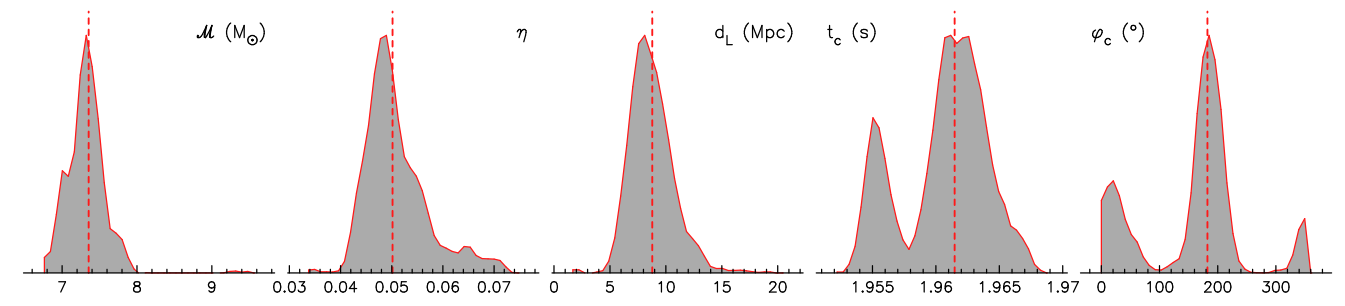

Figure 2: One-dimensional marginalised PDFs of a few selected parameters from our analysis of data set DS3. The vertical dashed lines indicate the median of each PDF.

\section{Conclusions}

We have developed the code SPINSPIRAL which can do a complete parameter analysis of the gravitational-wave signals from quasi-circular compact-binary inspirals. We presented an example of the analysis of software injections into both simulated Gaussian noise (DS1) and LIGO-detector data (DS2). We also presented an analysis of a data set containing no injection, but a "glitch" coincident in two LIGO interferometers (DS3). These examples demonstrate a remarkable similarity between the results obtained from a GW signal injected in Gaussian noise and a similar signal in detector data. The Bayes factors are also similar, where we note that our present technique for computing the Bayes factor yields estimates with significant variance, and more precise estimates should be possible in the future. In addition, we find that although the Markov chains in the analysis of a coincident glitch in LIGO data do not converge, the resulting PDFs could look remarkably consistent with a simulated GW signal. We plan to run our code on a very large number of coincident triggers from the LIGO Compact Binary Coalescence search pipeline (noise events that are somehow being registered as resembling a binary inspiral) in order to get a good sense of how to distinguish them from actual inspirals. We conclude that further, detailed investigations are necessary to ensure we can rely on the robustness of our tests.

\section{Acknowledgments}

The authors acknowledge a CITA National Fellowship to the UoA for MvdS, the NSF astronomy and astrophysics postdoctoral fellowship under the award AST-0901985 to IM, a NSF Gravitational Physics grant (PHY-0854790) to NC and the Max-PlanckSociety (CR). Computations were performed on the Fugu computer cluster funded by NSF MRI grant PHY-0619274 to VK.

\section{References}

Abadie J, Abbott B P \& al. 2010 ArXiv e-prints 1003.2480.

Abbott B, Abbott R \& al. 2009 a New Journal of Physics 11(7), 073032-+.

Abbott B P, Abbott R \& al. 2009b Phys. Rev. D 79(12), 122001.

Acernese F, Alshourbagy M \& al. 2008 Class. Quant. Grav. 25(18), 184001-+. 
Apostolatos T A, Cutler C, Sussman G J \& Thorne K S 1994 Phys. Rev. D 49, 6274-6297.

Atchadé Y F \& Rosenthal J S 2005 Bernoulli 11(5), 815-828.

Buonanno A, Chen Y \& Vallisneri M 2003 Phys. Rev. D 67(10), 104025.

Cutler C \& Flanagan É E 1994 Phys. Rev. D 49, 2658-2697.

Cutler C \& Thorne K S 2002 Proceedings of GR16, World Scientific, Singapore.

Earl D J \& Deem M W 2005 ArXiv e-prints physics/0508111.

Figueiredo M A T \& Jain A K 2002 IEEE Transactions on pattern analysis and machine intelligence 24(3), 381-396.

Gelman A, Carlin J B, Stern H \& Rubin D B 1997 Bayesian data analysis Chapman \& Hall, Boca Raton.

Gilks W R, Richardson S \& Spiegelhalter D J 1996 Markov chain Monte Carlo in practice Chapman \& Hall/CRC.

Hansmann U 1997 Chemical Physics Letters 281, 140-150.

Hukushima K \& Nemoto K 1996 Journal of the Physical Society of Japan 65, 1604.

Jaranowski P \& Krolak A 1994 Phys. Rev. D 49, 1723-1739.

LIGO Scientific Collaboration 2007 The LSC Algorithm Library. https://www.lsc-group.phys.uwm.edu/daswg/projects/lal.html.

Newton M A \& Raftery A E 1994 JRSS B 56(1), 3-48. http://www.jstor.org/stable/2346025.

O'Shaughnessy R, Kim C, Kalogera V \& Belczynski K 2008 ApJ 672, 479-488.

Poisson E \& Will C M 1995 Phys. Rev. D 52, 848-855.

Raymond V, Van der Sluys M V, Mandel I, Kalogera V, Röver C \& Christensen N 2009 Classical and Quantum Gravity 26(11), 114007.

Röver C 2007 Bayesian inference on astrophysical binary inspirals based on gravitational-wave measurements PhD thesis The University of Auckland. http://hdl.handle.net/2292/2356.

Röver C, Meyer R \& Christensen N 2006 Class. Quant. Grav. 23, 4895-4906.

Röver C, Meyer R \& Christensen N 2007 Phys. Rev. D 75(6), 062004.

Skilling J 2006 Bayesian Anal. 1(4), 833-859 (electronic).

Van den Broeck C \& Sengupta A S 2007 Class. Quant. Grav. 24, 1089-1113.

Van der Sluys M, Raymond V, Mandel I, Röver C, Christensen N, Kalogera V, Meyer R \& Vecchio A 2008a Class. Quant. Grav. 25(18), 184011.

Van der Sluys M V \& al. in preparation.

Van der Sluys M V, Röver C, Stroeer A, Raymond V, Mandel I, Christensen N, Kalogera V, Meyer R \& Vecchio A $2008 b$ ApJ 688, L61-L64.

Van Haasteren R 2009 ArXiv e-prints 0911.2150.

Veitch J \& Vecchio A 2008a Classical and Quantum Gravity 25(18), 184010.

Veitch J \& Vecchio A 2008 b Phys. Rev. D 78(2), 022001.

Veitch J \& Vecchio A 2009 ArXiv e-prints 0911.3820.

Willke B, Aufmuth P \& al. 2004 Class. Quant. Grav. 21, 417. 